\title{
Editorial
}

\section{Epidemic Processes on Complex Networks}

\author{
Lu-Xing Yang, ${ }^{1}$ Yong Deng, ${ }^{2}$ and Jose Roberto Castilho Piqueira ${ }^{3}$ \\ ${ }^{1}$ Faculty of Electrical Engineering, Mathematics and Computer Science, Delft University of Technology, 2600 GA Delft, Netherlands \\ ${ }^{2}$ School of Computer and Information Science, Southwest University, Chongqing 400715, China \\ ${ }^{3}$ Polytechnic School, University of Sao Paulo, 05508-900 Sao Paulo, SP, Brazil
}

Correspondence should be addressed to Lu-Xing Yang; ylx910920@gmail.com

Received 21 May 2017; Accepted 21 May 2017; Published 27 August 2017

Copyright (c) $2017 \mathrm{Lu}$-Xing Yang et al. This is an open access article distributed under the Creative Commons Attribution License, which permits unrestricted use, distribution, and reproduction in any medium, provided the original work is properly cited.

As a fascinating branch of the emerging network science, the mission of the epidemic dynamics on networks is to understand how objects spread in networks and thereby to work out cost-effective strategy for restraining undesirable objects or promoting desirable objects. This special issue contains ten excellent papers about this subject.

The basic function of the epidemic dynamics is to assess the risk of infectious diseases. J. Liu proposed an epidemic model with curing delay and explored its Hopf bifurcation properties. M. De la Sen et al. modeled the propagation of Ebola disease, determining the key factors affecting the global behavior of the model.

Another important role of the epidemic dynamics is to estimate the destructive effect of digital viruses. Z. Zhang et al. suggested a computer virus spreading model with two time delays (the curing delay and the vaccinating delay) and showed the possibility of a Hopf bifurcation. C. Gan et al. established and studied a digital virus model with nonlinear infection rates and external infection sources and developed a dynamic virus-inhibiting strategy. Q. Zhu and C. Cen presented an epidemic model in which different hosts in a network have separate security levels. Based on a novel individual-level virus-countermeasure interacting model, X. Zhang and C. Gan discussed the issue of how to optimally distribute countermeasures in a network. Based on an individual-level disruptive virus spreading model, J. Bi et al. developed a cost-effective dynamic strategy of restraining disruptive viruses.

An interesting application of the epidemic dynamics is to forecast the risk of attacks and the efficiency of security policies. Based on a new epidemic model and aiming at maximizing the utility of the entire system, Q. Shi et al. designed a cost-effective static detection strategy for defending against internal cyberattacks.

The epidemic dynamics can also be used to explore the way that opinions diffuse in social networks. Under an individual-level opinion spreading model, A. Lu uncovered the influence of the network structure on the converging time of opinion spreading.

For any undesirable transmissible object, the spectral radius minimization problem (SRMP) turns out to be crucial to contain its rampancy in a network. Y. Wu et al. studied the SRMP for a class of regular networks and obtained some results that would be instructive in developing heuristic algorithms for the SRMP.

\section{Acknowledgments}

We would like to express our great gratitude to all of the authors for their contributions and the reviewers for their serious evaluation of the papers submitted by valuable comments and timely feedback.

Lu-Xing Yang
Yong Deng
Jose Roberto Castilho Piqueira




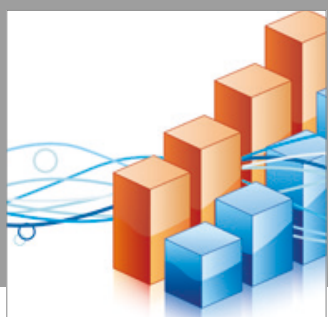

Advances in

Operations Research

vatersals

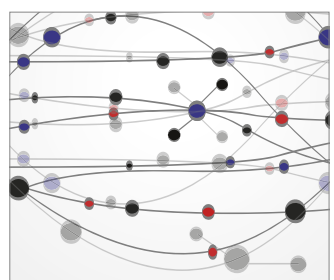

\section{The Scientific} World Journal
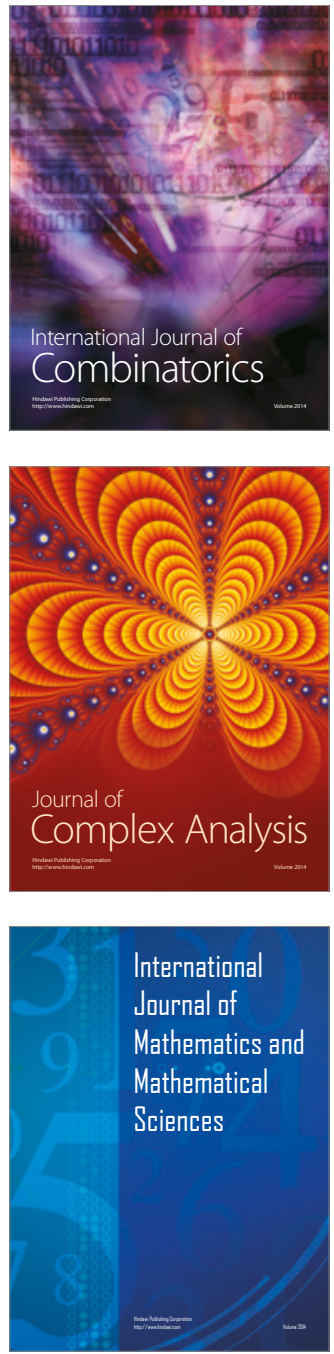
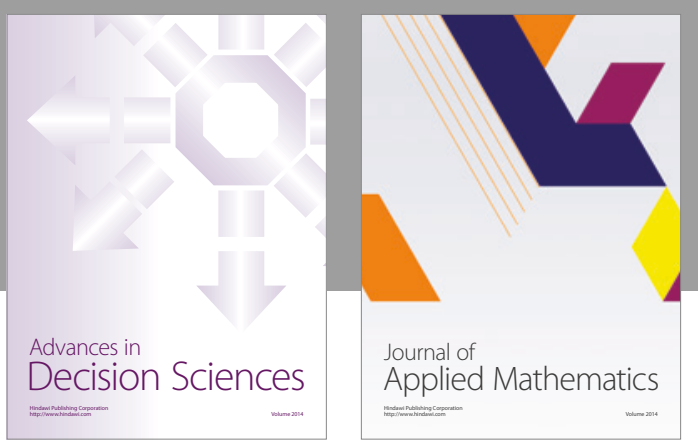

Algebra

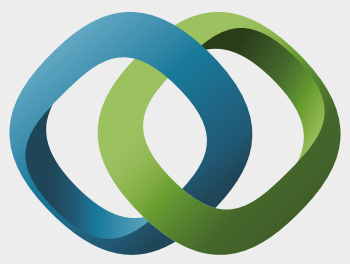

\section{Hindawi}

Submit your manuscripts at

https://www.hindawi.com
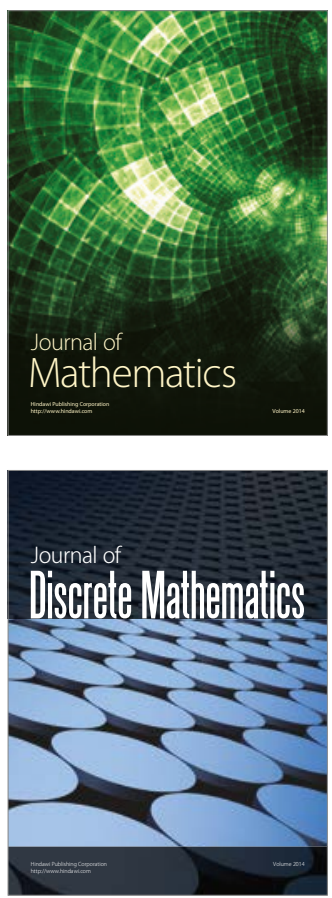

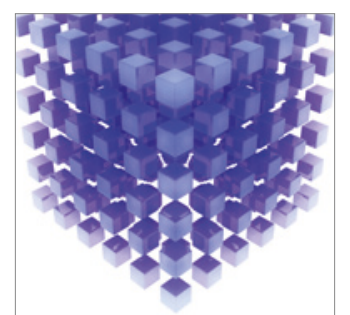

Mathematical Problems in Engineering
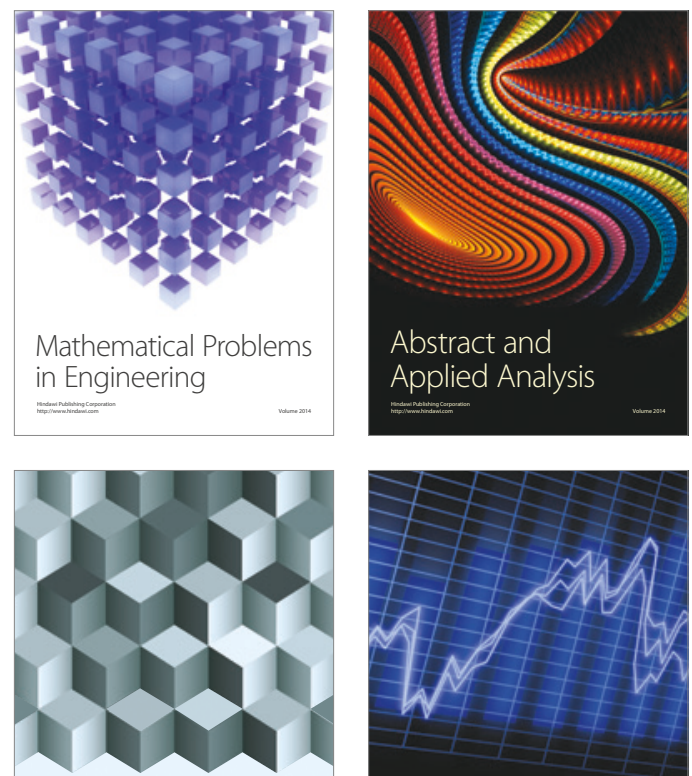

Journal of

Function Spaces

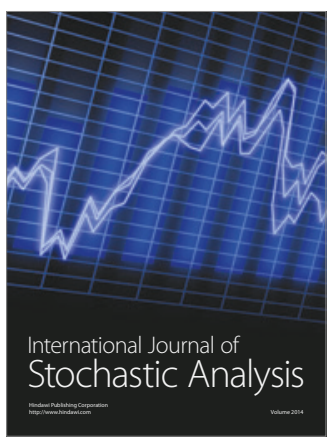

Probability and Statistics
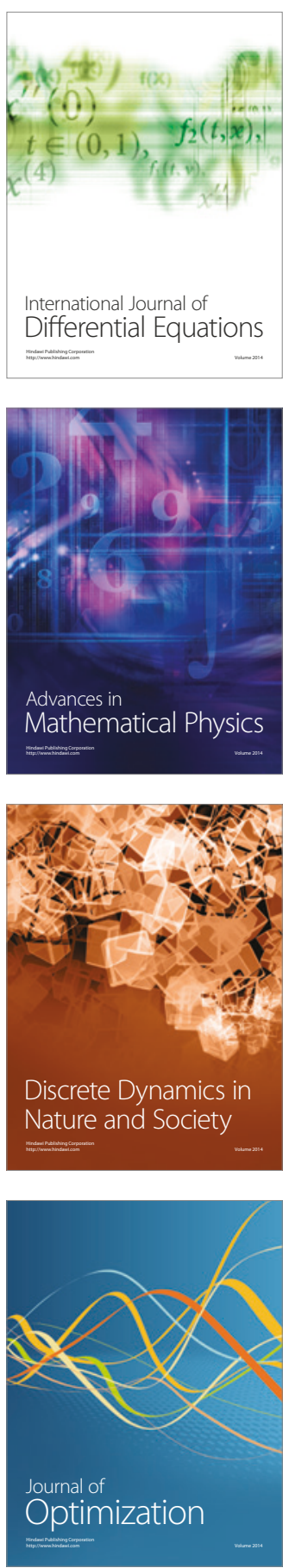